\title{
KEKONTINUAN FUNGSI PADA RUANG METRIK
}

Oleh:

\section{Cece Kustiawan}

Jurusan Pendidikan Matematika FPMIPA UPI, cecekustiawan@yahoo.com

\begin{abstract}
Pengertian fungsi di kalkulus adalah pemetaan dari himpunan bilangan real ke himpunan bilangan real dengan fungsi jaraknya adalah nilai mutlak. Pada makalah ini akan disajikan pengertian fungsi dari suatu ruang metrik ke ruang metrik yang lain yang fungsi jaraknya mungkin saja berbeda. Selanjutnya akan dibicarakan mengenai limit fungsi pada ruang metrik, kekontinuan fungsi pada ruang metrik, fungsi kontinu seragam pada ruang metrik, kekompakan fungsi pada ruang metrik, dan teorema-teorema yang berhubungan dengan hal tersebut.
\end{abstract}

Kata Kunci : Ruang Metrik, Limit Fungsi, Fungsi Kontinu, Fungsi Kompak.

Notion of a function in calculus is a mapping from the set of real numbers to the set of real numbers with absolute value it is. On this paper will be presented the notion of functions of a metric space into the other metric space with the functions of the distance is probably different. Next will be discussed regarding the limit of a function on a metric space, the continuous function on metric spaces, uniform continuity on the space metric, a metric space compactness function and theorems that relates to it.

Key words : Metric Space, Limit Of The Function, The Continuous Function, Compact Function.

\section{Pendahuluan}

Sebelum kita membicarakan Limit fungsi dan kekontinuan fungsi pada ruang metrik terlebih dahulu kita bahas mengenai definisi metrik, definisi persekitaran pada ruang metrik, definisi titik limit pada ruang metrik, definisi himpunan terbuka pada ruang metrik, pengertian selimut terbuka pada ruang metrik, dan definisi kompak pada ruang metrik.

Misalkan $\mathrm{X}$ himpunan yang tidak kosong. Fungsi $\mathrm{d}: \mathrm{X} \mathrm{x} \mathrm{X} \rightarrow \mathrm{R}$ disebut fungsi metrik (fungsi jarak) jika untuk setiap $p, q \in X$ berlaku :

(i) $\mathrm{d}(\mathrm{p}, \mathrm{q}) \geq 0$

$$
d(p, q)=0 \Leftrightarrow p=q
$$

(ii) $\mathrm{d}(\mathrm{p}, \mathrm{q})=\mathrm{d}(\mathrm{q}, \mathrm{p})$

(iii) $\mathrm{d}(\mathrm{p}, \mathrm{q}) \leq \mathrm{d}(\mathrm{p}, \mathrm{r})+\mathrm{d}(\mathrm{r}, \mathrm{q}), \forall \mathrm{r} \in \mathrm{X}$.

Himpunan $\mathrm{X}$ dengan fungsi metrik d disebut ruang metrik dan ditulis dengan notasi $(\mathrm{X}, \mathrm{d})$ atau $\mathrm{X}$ saja. 
Misalkan (X,d) ruang metrik. Persekitaran (neighborhood) dari titik p ditulis dengan notasi $\mathrm{N}_{\mathrm{r}}(\mathrm{p}), \mathrm{r}>0$ dan didefinisikan sebagai berikut;

$$
\mathrm{N}_{\mathrm{r}}(\mathrm{p})=\{\mathrm{q} \in X: \mathrm{d}(\mathrm{p}, \mathrm{q})<\mathrm{r}\} .
$$

r disebut jari-jari dari persekitaran $\mathrm{N}_{\mathrm{r}}(\mathrm{p})$.

Misalkan $(\mathrm{X}, \mathrm{d})$ ruang metrik dan $\mathrm{E} \subseteq \mathrm{X}$. Titik p disebut titik limit dari $\mathrm{E}$ jika setiap persekitaran $\mathrm{N}_{\mathrm{r}}(\mathrm{p})$ memuat titik anggota $\mathrm{E}$ yang tidak sama dengan $\mathrm{p}$. Equivalen dengan, $p$ disebut titik limit dari $E$ jika setiap persekitaran $N_{r}(p)$ ada $\mathrm{q} \in \mathrm{N}_{\mathrm{r}}(\mathrm{p}) \cap \mathrm{E} \backslash\{\mathrm{p}\}$ atau equivalen dengan, $\mathrm{p}$ disebut titik limit dari $\mathrm{E}$ jika setiap persekitaran $\mathrm{N}_{\mathrm{r}}(\mathrm{p})$ berlaku $\mathrm{N}_{\mathrm{r}}(\mathrm{p}) \cap \mathrm{E} \backslash\{\mathrm{p}\} \neq \phi$.

Misalkan (X,d) ruang metrik dan $\mathrm{E} \subseteq \mathrm{X} . \mathrm{p} \in \mathrm{E}$ disebut titik dalam dari $\mathrm{E}$ jika ada persekitaran $\mathrm{N}_{\mathrm{r}}(\mathrm{p})$ sehingga $\mathrm{p} \in \mathrm{N}_{\mathrm{r}}(\mathrm{p}) \subset \mathrm{E}$. Selanjutnya $\mathrm{E}$ disebut himpunan terbuka jika setiap anggota $\mathrm{E}$ merupakan titik dalam dari E. Jadi $\mathrm{E}$ disebut himpunan terbuka jika setiap $\mathrm{p} \in \mathrm{E}$ ada persekitaran $\mathrm{N}_{\mathrm{r}}(\mathrm{p})$ sehingga $\mathrm{p} \in \mathrm{N}_{\mathrm{r}}(\mathrm{p}) \subset \mathrm{E}$.

Selimut terbuka himpunan $\mathrm{E}$ dalam ruang metrik (X,d) adalah keluarga himpunan terbuka $\left\{G_{\alpha}\right\}$ di $X$ sehingga $E \subset \bigcup_{\alpha} G_{\alpha}$. Selanjutnya Himpunan $K$ dalam ruang metrik $(\mathrm{X}, \mathrm{d})$ dikatakan kompak jika setiap selimut terbuka untuk K memuat selimut bagian berhingganya untuk $\mathrm{K}$.

\section{Pembahasan}

\section{Limit Fungsi}

\section{Definisi 1.}

Misalkan $\mathrm{X}$ dan $\mathrm{Y}$ ruang metrik, $\mathrm{E} \subset \mathrm{X}, \mathrm{f}: \mathrm{E} \rightarrow \mathrm{Y}$ dan $\mathrm{p}$ titik limit $\mathrm{E}$. $\lim _{x \rightarrow p} f(x)=q$ jika dan hanya jika untuk setiap $\varepsilon>0$ terdapat $\delta>0$ sehingga untuk setiap $\mathrm{x} \in \mathrm{E}$ dengan $0<\mathrm{d}_{\mathrm{x}}(\mathrm{x}, \mathrm{p})<\delta$ berlaku $\mathrm{d}_{\mathrm{Y}}(\mathrm{f}(\mathrm{x}), \mathrm{q})<\varepsilon$.

Catatan :

$\mathrm{p} \in \mathrm{X}$ tetapi $\mathrm{p}$ tidak perlu anggota $\mathrm{E}$.

Walaupun $\mathrm{p} \in \mathrm{E}$ mungkin saja $\mathrm{f}(\mathrm{p}) \neq \lim _{\mathrm{x} \rightarrow \mathrm{p}} \mathrm{f}(\mathrm{x})$ artinya ia tidak kontinu di $\mathrm{p}$.

$\mathrm{d}_{\mathrm{x}}$ adalah jarak pada ruang metrik $\mathrm{X}$ dan $\mathrm{d}_{\mathrm{Y}}$ adalah jarak pada ruang metrik $\mathrm{Y}$. Jika $\mathrm{X}$ atau $\mathrm{Y}$ diganti oleh $\mathrm{R}$, maka $\mathrm{d}_{\mathrm{x}}, \mathrm{d}_{\mathrm{Y}}$ berarti nilai mutlak. 


\section{Teorema 2.}

Misalkan $\mathrm{X}$ dan $\mathrm{Y}$ ruang metrik, $\mathrm{E} \subset \mathrm{X}, \mathrm{f}: \mathrm{E} \rightarrow \mathrm{Y}$ dan $\mathrm{p}$ titik limit $\mathrm{E}$. $\lim _{x \rightarrow p} f(x)=q$ jika dan hanya jika $\lim _{n \rightarrow \infty} f\left(p_{n}\right)=q$ untuk setiap barisan $\left\{p_{n}\right\}$ didalam E dengan $\mathrm{p}_{\mathrm{n}} \neq \mathrm{p}, \forall \mathrm{n} \in \mathrm{N}$ dan $\lim _{\mathrm{n} \rightarrow \infty} \mathrm{p}_{\mathrm{n}}=\mathrm{p}$.

\section{Bukti :}

$(\Rightarrow)$ Diketahui $p$ titik limit $E$ dan $\lim _{x \rightarrow p} f(x)=q$. Akan diperlihatkan $\lim _{n \rightarrow \infty} f\left(p_{n}\right)=q$ untuk setiap barisan $\left\{\mathrm{p}_{\mathrm{n}}\right\}$ didalam $\mathrm{E}, \mathrm{p}_{\mathrm{n}} \neq \mathrm{p}, \forall \mathrm{n} \in \mathrm{N}$ dan $\lim _{\mathrm{n} \rightarrow \infty} \mathrm{p}_{\mathrm{n}}=\mathrm{p}$. Diberikan $\varepsilon>0$ sebarang dan barisan $\left\{\mathrm{p}_{\mathrm{n}}\right\}$ didalam $\mathrm{E}, \mathrm{p}_{\mathrm{n}} \neq \mathrm{p}, \forall \mathrm{n} \in \mathrm{N}$ dan $\lim _{\mathrm{n} \rightarrow \infty} \mathrm{p}_{\mathrm{n}}=\mathrm{p}$.

Karena $\lim _{\mathrm{x} \rightarrow \mathrm{p}} \mathrm{f}(\mathrm{x})=\mathrm{q}$ maka $\forall \varepsilon>0$ terdapat $\delta>0$ sehingga untuk setiap $\mathrm{x} \in \mathrm{E}$ dengan $0<\mathrm{d}_{\mathrm{X}}(\mathrm{x}, \mathrm{p})<\delta$ berlaku $\mathrm{d}_{\mathrm{Y}}(\mathrm{f}(\mathrm{x}), \mathrm{q})<\varepsilon$. Karena $\lim _{\mathrm{n} \rightarrow \infty} \mathrm{p}_{\mathrm{n}}=\mathrm{p}$ maka untuk $\delta>0$ di atas ada $\mathrm{n}_{0} \in \mathrm{N}$ sehingga untuk setiap $\mathrm{n} \geq \mathrm{n}_{0}$ berlaku $0<\mathrm{d}_{\mathrm{X}}\left(\mathrm{p}_{\mathrm{n}}, \mathrm{p}\right)<\delta$. Akibatnya $\mathrm{d}_{\mathrm{Y}}\left(\mathrm{f}\left(\mathrm{p}_{\mathrm{n}}\right), \mathrm{q}\right)<\varepsilon$. Jadi untuk setiap $\varepsilon>0$ terdapat $\mathrm{n}_{0} \in \mathrm{N}$ sehingga untuk setiap $\mathrm{n} \geq \mathrm{n}_{0}$ berlaku $\mathrm{d}_{\mathrm{Y}}\left(\mathrm{f}\left(\mathrm{p}_{\mathrm{n}}\right), \mathrm{q}\right)<\varepsilon$. Hal ini berarti $\lim _{n \rightarrow \infty} f\left(p_{n}\right)=q$.

$(\Leftarrow)$ Andaikan $\lim _{\mathrm{x} \rightarrow \mathrm{p}} \mathrm{f}(\mathrm{x}) \neq \mathrm{q}$ maka terdapat $\varepsilon>0$ sehingga untuk setiap $\delta>0$ ada $\mathrm{x} \in \mathrm{E}$ dengan $0<\mathrm{d}_{\mathrm{X}}(\mathrm{x}, \mathrm{p})<\delta$ tetapi $\mathrm{d}_{\mathrm{Y}}(\mathrm{f}(\mathrm{x}), \mathrm{q}) \geq \varepsilon$.

Karena $\lim _{\mathrm{n} \rightarrow \infty} \mathrm{p}_{\mathrm{n}}=\mathrm{p}$ maka untuk $\delta=\frac{1}{\mathrm{n}}>0, \mathrm{n} \in \mathrm{N}$ ada $\mathrm{n}_{0} \in \mathrm{N}$ sehingga untuk setiap $\mathrm{n} \geq \mathrm{n}_{0}$ berlaku $0<\mathrm{d}_{\mathrm{X}}\left(\mathrm{p}_{\mathrm{n}}, \mathrm{p}\right)<\delta$ tetapi $\mathrm{d}_{\mathrm{Y}}\left(\mathrm{f}\left(\mathrm{p}_{\mathrm{n}}\right), \mathrm{q}\right) \geq \varepsilon$. Hal ini berarti $\lim _{x \rightarrow p} f\left(p_{n}\right) \neq q$ yang kontradiksi dengan yang diketahui bahwa $\lim _{n \rightarrow \infty} f\left(p_{n}\right)=q$. Jadi pengandaian salah dan haruslah $\lim _{x \rightarrow p} f(x)=q$.

\section{Akibat 3.}

Jika f mempunyai titik limit di p maka limitnya tunggal

\section{Bukti :}

Misalkan $\lim _{\mathrm{x} \rightarrow \mathrm{p}} \mathrm{f}(\mathrm{x})=\mathrm{L}_{1}$ dan $\lim _{\mathrm{x} \rightarrow \mathrm{p}} \mathrm{f}(\mathrm{x})=\mathrm{L}_{2}$. Akan diperlihatkan $\mathrm{L}_{1}=\mathrm{L}_{2}$.

Diberikan $\varepsilon>0$ sebarang. 


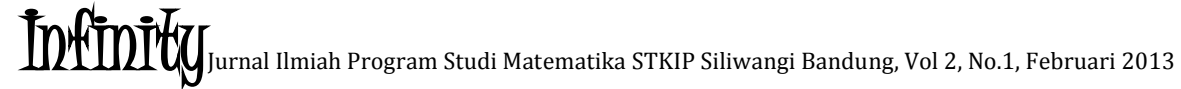

Karena $\lim _{x \rightarrow p} f(x)=L_{1}$, maka terdapat $\delta_{1}>0$ sehingga untuk setiap $x \in E$ dengan $0<\mathrm{d}_{\mathrm{x}}(\mathrm{x}, \mathrm{p})<\delta_{1}$ berlaku $\mathrm{d}_{\mathrm{Y}}\left(\mathrm{f}(\mathrm{x}), \mathrm{L}_{1}\right)<\frac{\varepsilon}{2}$. Dipihak lain karena $\lim _{\mathrm{x} \rightarrow \mathrm{p}} \mathrm{f}(\mathrm{x})=\mathrm{L}_{2}$, maka terdapat $\delta_{2}>0$ sehingga untuk setiap $\mathrm{x} \in \mathrm{E}$ dengan $0<\mathrm{d}_{\mathrm{x}}(\mathrm{x}, \mathrm{p})<\delta_{2}$ berlaku $\mathrm{d}_{\mathrm{Y}}\left(\mathrm{f}(\mathrm{x}), \mathrm{L}_{2}\right)<\frac{\varepsilon}{2}$. Jika diambil $\delta=\min \left\{\delta_{1}, \delta_{2}\right\}$, maka untuk setiap $\mathrm{x} \in \mathrm{E}$ dengan $0<\mathrm{d}_{\mathrm{x}}(\mathrm{x}, \mathrm{p})<\delta$ berlaku $\mathrm{d}_{\mathrm{Y}}\left(\mathrm{f}(\mathrm{x}), \mathrm{L}_{1}\right)<\frac{\varepsilon}{2}$ dan $\mathrm{d}_{\mathrm{Y}}\left(\mathrm{f}(\mathrm{x}), \mathrm{L}_{2}\right)<\frac{\varepsilon}{2}$.

Akibatnya $0<\mathrm{d}_{\mathrm{Y}}\left(\mathrm{L}_{1}, \mathrm{~L}_{2}\right) \leq \mathrm{d}_{\mathrm{Y}}\left(\mathrm{L}_{1}, \mathrm{f}(\mathrm{x})\right)+\mathrm{d}_{\mathrm{Y}}\left(\mathrm{f}(\mathrm{x}), \mathrm{L}_{2}\right)<\frac{\varepsilon}{2}+\frac{\varepsilon}{2}=\varepsilon$.

Karena $\varepsilon>0$ sebarang, maka $\mathrm{d}_{\mathrm{Y}}\left(\mathrm{L}_{1}, \mathrm{~L}_{2}\right)=0$ atau $\mathrm{L}_{1}=\mathrm{L}_{2}$.

\section{Definisi 4.}

Jika $f$ dan $g$ suatu fungsi yang terdefinisi pada $E$ dan $\lambda$ suatu konstanta, maka :

(i) $(\mathrm{f}+\mathrm{g})(\mathrm{x})=\mathrm{f}(\mathrm{x})+\mathrm{g}(\mathrm{x})$

(ii) $(\mathrm{f} . \mathrm{g})(\mathrm{x})=\mathrm{f}(\mathrm{x}) \cdot \mathrm{g}(\mathrm{x})$

(iii) $(\lambda \mathrm{f})(\mathrm{x})=\lambda . \mathrm{f}(\mathrm{x})$

\section{Teorema 5.}

Misalkan $\mathrm{X}$ ruang metrik, $\mathrm{E} \subset \mathrm{X}, \mathrm{p}$ titik limit $\mathrm{E}$, fungsi $\mathrm{f}$ dan $\mathrm{g}$ terdefinisi pada E. Jika $\lim _{x \rightarrow p} f(x)=A$ dan $\lim _{x \rightarrow p} g(x)=B$, maka :

(i) $\lim _{x \rightarrow p}(f+g)(x)=A+B$

(ii) $\lim _{x \rightarrow p}(f . g)(x)=A \cdot B$

(iii) $\lim _{x \rightarrow p}\left(\frac{f}{g}\right)(x)=\frac{A}{B}$, asalkan $\mathrm{g}(\mathrm{x}) \neq 0$ untuk $\forall x \in \mathrm{X}$ dan $\mathrm{B} \neq 0$.

\section{Bukti :}

(i) Diberikan $\varepsilon>0$ sebarang.

Karena $\lim _{\mathrm{x} \rightarrow \mathrm{p}} \mathrm{f}(\mathrm{x})=\mathrm{A}$, maka terdapat $\delta_{1}>0$ sehingga untuk setiap $\mathrm{x} \in \mathrm{E}$ dengan $0<\mathrm{d}_{\mathrm{x}}(\mathrm{x}, \mathrm{p})<\delta_{1}$ berlaku $\mathrm{d}_{\mathrm{Y}}(\mathrm{f}(\mathrm{x}), \mathrm{A})<\frac{\varepsilon}{2}$. Dipihak lain karena $\lim _{x \rightarrow p} g(x)=B$, maka terdapat $\delta_{2}>0$ sehingga untuk setiap $x \in E$ dengan $0<\mathrm{d}_{\mathrm{x}}(\mathrm{x}, \mathrm{p})<\delta_{2}$ berlaku $\mathrm{d}_{\mathrm{Y}}(\mathrm{g}(\mathrm{x}), \mathrm{B})<\frac{\varepsilon}{2}$. Jika diambil $\delta=\min \left\{\delta_{1}, \delta_{2}\right\}$, maka untuk setiap $\mathrm{x} \in \mathrm{E}$ dengan $0<\mathrm{d}_{\mathrm{x}}(\mathrm{x}, \mathrm{p})<\delta$ berlaku $\mathrm{d}_{\mathrm{Y}}(\mathrm{f}(\mathrm{x}), \mathrm{A})<\frac{\varepsilon}{2}$ 


\section{Infíñit ty}

dan $\mathrm{d}_{\mathrm{Y}}(\mathrm{g}(\mathrm{x}), \mathrm{B})<\frac{\varepsilon}{2}$. Akibatnya

$\mathrm{d}_{\mathrm{Y}}(\mathrm{f}(\mathrm{x})+\mathrm{g}(\mathrm{x}), \mathrm{A}+\mathrm{B}) \leq \mathrm{d}_{\mathrm{Y}}(\mathrm{f}(\mathrm{x}), \mathrm{A})+\mathrm{d}_{\mathrm{Y}}(\mathrm{g}(\mathrm{x}), \mathrm{B})<\frac{\varepsilon}{2}+\frac{\varepsilon}{2}=\varepsilon$.

Jadi untuk setiap $\varepsilon>0$ ada $\delta>0$ sehingga jika $x \in E$ dan $0<\mathrm{d}_{\mathrm{x}}(\mathrm{x}, \mathrm{p})<\delta$ berlaku $d_{Y}(f(x)+g(x), A+B)<\varepsilon$. Hal ini berarti $\lim _{x \rightarrow p}(f+g)(x)=A+B$.

(ii) Diberikan $\varepsilon>0$ sebarang.

Karena $\lim _{x \rightarrow p} f(x)=A$, maka berdasarkan teorema 2, $\lim _{n \rightarrow \infty} f\left(p_{n}\right)=A$ untuk setiap barisan $\left\{p_{n}\right\}$ didalam $E$ dengan $p_{n} \neq p, \forall n \in N$ dan $\lim _{n \rightarrow \infty} p_{n}=p$. Begitu juga karena $\lim _{x \rightarrow p} g(x)=B$, maka $\lim _{n \rightarrow \infty} g\left(p_{n}\right)=B$ untuk setiap barisan $\left\{p_{n}\right\}$ didalam $\mathrm{E}$ dengan $\mathrm{p}_{\mathrm{n}} \neq \mathrm{p}, \forall \mathrm{n} \in \mathrm{N}$ dan $\lim _{\mathrm{n} \rightarrow \infty} \mathrm{p}_{\mathrm{n}}=\mathrm{p}$. Jadi untuk setiap barisan $\left\{\mathrm{p}_{\mathrm{n}}\right\}$ didalam $\mathrm{E}$ dengan $\mathrm{p}_{\mathrm{n}} \neq \mathrm{p}, \quad \forall \mathrm{n} \in \mathrm{N}$ dan $\quad \lim _{n \rightarrow \infty} p_{n}=p$ diperoleh $\lim _{x \rightarrow p}(f g)(x)=\lim _{x \rightarrow p} f(x) \lim _{x \rightarrow p} g(x)=\lim _{n \rightarrow \infty} f\left(p_{n}\right) \lim _{n \rightarrow \infty} g\left(p_{n}\right)=A B$.

(iii) analog dengan (ii)

\section{Fungsi Kontinu}

Selanjutnya kita bicara kekontinuan fungsi di ruang metrik. Untuk menentukan apakah suatu fungsi kontinu atau tidak, kita bisa menggunakan definisi atau boleh juga menggunakan teorema, sebagaimana dijelaskan berikut ini.

\section{Definisi 6}

Misalkan $\mathrm{X}$ dan $\mathrm{Y}$ ruang metrik, $\mathrm{E} \subset \mathrm{X}, \mathrm{p} \in \mathrm{E}, \mathrm{f}: \mathrm{E} \rightarrow \mathrm{Y}$.

f kontinu di $\mathrm{p} \Leftrightarrow$ untuk setiap $\varepsilon>0$ ada $\delta>0$ sehingga untuk setiap $\mathrm{x} \in \mathrm{E}$ dengan $\mathrm{d}_{\mathrm{X}}(\mathrm{x}, \mathrm{p})<\delta$ berlaku $\mathrm{d}_{\mathrm{Y}}(\mathrm{f}(\mathrm{x}), \mathrm{f}(\mathrm{p}))<\varepsilon$.

Jika $\mathrm{f}$ kontinu di setiap titik $\mathrm{E}$, maka dikatakan $\mathrm{f}$ kontinu pada $\mathrm{E}$.

\section{Teorema 7}

Misalkan $\mathrm{X}$ dan $\mathrm{Y}$ ruang metrik, $\mathrm{E} \subset \mathrm{X}, \mathrm{p}$ titik limit dari $\mathrm{E}$ dan $\mathrm{f}: \mathrm{E} \rightarrow \mathrm{Y}$. f kontinu di $\mathrm{p} \Leftrightarrow \lim _{x \rightarrow p} f(x)=f(p)$.

\section{Bukti :}

$(\Rightarrow)$ Diketahui f kontinu di p, akan diperlihatkan $\lim _{x \rightarrow p} f(x)=f(p)$. 
Karena f kontinu di p, maka untuk setiap $\varepsilon>0$ ada $\delta>0$ sehingga untuk setiap $\mathrm{x} \in \mathrm{E}$ dengan $\mathrm{d}_{\mathrm{X}}(\mathrm{x}, \mathrm{p})<\delta$ berlaku $\mathrm{d}_{\mathrm{Y}}(\mathrm{f}(\mathrm{x}), \mathrm{f}(\mathrm{p}))<\varepsilon$. Dipihak lain karena $\mathrm{p}$ titik limit $\mathrm{E}$, maka untuk $\delta>0$ tersebut (atau untuk $\mathrm{N}_{\delta}(\mathrm{p})$ ) ada $\mathrm{x} \in \mathrm{N}_{\delta}(\mathrm{p}) \cap \mathrm{El}\{\mathrm{p}\}$. Berarti ada $\mathrm{x} \in \mathrm{E}, 0<\mathrm{d}_{\mathrm{X}}(\mathrm{x}, \mathrm{p})<\delta$ dan berakibat $\mathrm{d}_{\mathrm{Y}}(\mathrm{f}(\mathrm{x}), \mathrm{f}(\mathrm{p}))<\varepsilon$. Hal ini berarti $\lim _{x \rightarrow p} f(x)=f(p)$.

$(\Leftarrow)$ Diketahui $\lim _{x \rightarrow p} f(x)=f(p)$, akan diperlihatkan f kontinu di p.

Karena $\lim _{x \rightarrow p} f(x)=f(p)$, maka untuk setiap $\varepsilon>0$ ada $\delta>0$ sehingga untuk setiap $\mathrm{x} \in \mathrm{E}$ dengan $0<\mathrm{d}_{\mathrm{X}}(\mathrm{x}, \mathrm{p})<\delta$ berlaku $\mathrm{d}_{\mathrm{Y}}(\mathrm{f}(\mathrm{x}), \mathrm{f}(\mathrm{p}))<\varepsilon$. Tetapi untuk $\mathrm{x} \in \mathrm{E}$ dengan $\mathrm{d}_{\mathrm{X}}(\mathrm{x}, \mathrm{p})<\delta$ tetap berlaku $\mathrm{d}_{\mathrm{Y}}(\mathrm{f}(\mathrm{x}), \mathrm{f}(\mathrm{p}))<\varepsilon$ yang berarti f kontinu di $\mathrm{p}$.

\section{Teorema 8 (Komposisi Fungsi)}

Misalkan $X, Y$ dan $Z$ ruang metrik, $E \subset X, f(E) \subset Y, f: E \rightarrow Y, g: f(E) \rightarrow Z$. Jika f kontinu di $\mathrm{p} \in \mathrm{E}$ dan $\mathrm{g}$ kontinu di $\mathrm{f}(\mathrm{p}) \in \mathrm{f}(\mathrm{E})$, maka $\mathrm{g}_{\mathrm{o}} \mathrm{f}$ kontinu di $\mathrm{p}$.

\section{Bukti :}

Diberikan $\varepsilon>0$ sebarang.

Karena g kontinu di $\mathrm{f}(\mathrm{p})$, maka ada $\delta>0$ sehingga untuk setiap y $\in \mathrm{E}$ dengan $\mathrm{d}_{\mathrm{Y}}(\mathrm{y}, \mathrm{f}(\mathrm{p}))<\delta$ berlaku $\mathrm{d}_{\mathrm{Z}}(\mathrm{g}(\mathrm{y}), \mathrm{g}(\mathrm{f}(\mathrm{p})))<\varepsilon$. Dipihak lain karena $\mathrm{f}$ kontinu di $\mathrm{p}$, maka untuk $\delta>0$ tersebut, ada $\eta>0$ sehingga untuk setiap $x \in E$ dengan $d_{X}(x, p)<\eta$ berlaku $\mathrm{d}_{\mathrm{Y}}(\mathrm{f}(\mathrm{x}), \mathrm{f}(\mathrm{p}))<\delta$. Akibatnya $\mathrm{d}_{\mathrm{Z}}(\mathrm{g}(\mathrm{f}(\mathrm{x})), \mathrm{g}(\mathrm{f}(\mathrm{p})))<\varepsilon$.

Jadi untuk setiap $\varepsilon>0$, ada $\eta>0$ sehingga untuk setiap $x \in E$ dengan $d_{x}(x, p)<\eta$ berlaku $d_{Z}(g(f(x)), g(f(p)))<\varepsilon$. Hal ini berarti gontinu di $p$.

\section{Teorema 9}

Misalkan $\mathrm{X}$ dan Y ruang metrik, $\mathrm{f}: \mathrm{X} \rightarrow \mathrm{Y}$.

f kontinu pada $X \Leftrightarrow \mathrm{f}^{-1}(\mathrm{~V})$ terbuka didalam $X$, untuk setiap himpunan terbuka $\mathrm{V}$ didalam $\mathrm{Y}$.

\section{Bukti :}

$(\Rightarrow)$ Diketahui f kontinu pada X. Diambil sebarang himpunan terbuka V didalam $Y$, akan diperlihatkan $\mathrm{f}^{-1}(\mathrm{~V})$ terbuka didalam $\mathrm{X}$.

Misalkan $\mathrm{p} \in \mathrm{f}^{-1}(\mathrm{~V})$, maka $\mathrm{f}(\mathrm{p}) \in \mathrm{V}$ dan karena $\mathrm{V}$ terbuka didalam $\mathrm{Y}$, maka $\mathrm{f}(\mathrm{p})$ merupakan titik dalam dari $\mathrm{V}$, artinya ada $\varepsilon>0$ sehingga persekitaran $\mathrm{N}_{\varepsilon}(\mathrm{f}(\mathrm{p})) \subset \mathrm{V}$. Jadi jika $\mathrm{y} \in \mathrm{N}_{\varepsilon}(\mathrm{f}(\mathrm{p}))$, maka $\mathrm{d}_{\mathrm{Y}}(\mathrm{y}, \mathrm{f}(\mathrm{p}))<\varepsilon$. Dilain pihak karena $\mathrm{f}$ kontinu di $\mathrm{p}$, maka untuk $\varepsilon>0$ tersebut ada $\delta>0$ sehingga jika $\mathrm{d}_{\mathrm{X}}(\mathrm{x}, \mathrm{p})<\delta$ berlaku $\mathrm{d}_{\mathrm{Y}}(\mathrm{f}(\mathrm{x}), \mathrm{f}(\mathrm{p}))<\varepsilon$. 
Dengan kata lain untuk $\varepsilon>0$ tersebut ada $\delta>0$ sehingga jika $\mathrm{x} \in \mathrm{N}_{\delta}(\mathrm{p})$ berlaku $\mathrm{f}(\mathrm{x}) \in \mathrm{N}_{\varepsilon}(\mathrm{f}(\mathrm{p})) \subset \mathrm{V}$ atau $\mathrm{x} \in \mathrm{f}^{-1}(\mathrm{~V})$, berarti $\mathrm{N}_{\delta}(\mathrm{p}) \subset \mathrm{f}^{-1}(\mathrm{~V})$. Jadi untuk setiap $\mathrm{p} \in \mathrm{f}^{-1}$ $(\mathrm{V})$, ada $\delta>0$ sehingga persekitaran $\mathrm{N}_{\delta}(\mathrm{p}) \subset \mathrm{f}^{-1}(\mathrm{~V})$ yang berarti $\mathrm{f}^{-1}(\mathrm{~V})$ terbuka.

$(\Leftarrow)$ Diketahui $\mathrm{f}^{-1}(\mathrm{~V})$ terbuka didalam $\mathrm{X}$ untuk setiap himpunan terbuka $\mathrm{V}$ didalam $\mathrm{Y}$, akan diperlihatkan $\mathrm{f}$ kontinu pada $\mathrm{X}$.

Diambil $\mathrm{p} \in \mathrm{X}$ dan $\varepsilon>0$ sebarang, maka $\mathrm{f}(\mathrm{p}) \in \mathrm{Y}$.

Misalkan $\mathrm{V}=\left\{\mathrm{y} \in \mathrm{Y}: \mathrm{d}_{\mathrm{Y}}(\mathrm{y}, \mathrm{f}(\mathrm{p}))<\varepsilon\right\}$, maka $\mathrm{V}$ merupakan himpunan terbuka didalam $\mathrm{Y}$ dan berdasarkan yang diketahui $\mathrm{f}^{-1}(\mathrm{~V})$ terbuka didalam $\mathrm{X}$. Akibatnya ada $\delta>0$ sehingga persekitaran $\mathrm{N}_{\delta}(\mathrm{p}) \subset \mathrm{f}^{-1}(\mathrm{~V})$, berarti jika $\mathrm{x} \in \mathrm{N}_{\delta}(\mathrm{p})$ maka $\mathrm{x} \in \mathrm{f}^{-1}(\mathrm{~V})$ atau jika $\mathrm{d}_{\mathrm{X}}(\mathrm{x}, \mathrm{p})<\delta$ maka $\mathrm{f}(\mathrm{x}) \in \mathrm{V}$ atau jika $\mathrm{d}_{\mathrm{X}}(\mathrm{x}, \mathrm{p})<\delta$ maka $\mathrm{d}_{\mathrm{Y}}(\mathrm{f}(\mathrm{x}), \mathrm{f}(\mathrm{p}))<\varepsilon$. Jadi untuk setiap $\varepsilon>0$ ada $\delta>0$ sehingga jika $\mathrm{d}_{\mathrm{X}}(\mathrm{x}, \mathrm{p})<\delta$ maka $\mathrm{d}_{\mathrm{Y}}(\mathrm{f}(\mathrm{x}), \mathrm{f}(\mathrm{p}))<\varepsilon$. Hal ini berarti $f$ kontinu di $p$ dan karena $p \in X$ sebarang maka $f$ kontinu pada $X$.

\section{Akibat 10}

Misalkan $\mathrm{X}$ dan $\mathrm{Y}$ ruang metrik, $\mathrm{f}: \mathrm{X} \rightarrow \mathrm{Y}$.

$\mathrm{f}$ kontinu pada $\mathrm{X} \Leftrightarrow \mathrm{f}^{-1}(\mathrm{~W})$ tertutup didalam $\mathrm{X}$, untuk setiap himpunan tertutup $\mathrm{W}$ didalam $\mathrm{Y}$.

\section{Bukti :}

$(\Rightarrow)$ Diketahui f kontinu pada X. Diambil sebarang himpunan tertutup W didalam $\mathrm{Y}$, akan diperlihatkan $\mathrm{f}^{-1}(\mathrm{~W})$ tertutup didalam $\mathrm{X}$.

$\mathrm{W}$ tertutup didalam $\mathrm{Y}$ maka $\mathrm{W}^{\mathrm{c}}$ terbuka didalam $\mathrm{Y}$. Karena $\mathrm{f}$ kontinu pada $\mathrm{X}$ maka berdasarkan teorema $9, \mathrm{f}^{-1}\left(\mathrm{~W}^{\mathrm{c}}\right)$ terbuka didalam $\mathrm{X}$.

Dipihak lain $\mathrm{f}^{-1}\left(\mathrm{~W}^{\mathrm{c}}\right)=\left(\mathrm{f}^{-1}(\mathrm{~W})\right)^{\mathrm{c}}$ atau $\left(\mathrm{f}^{-1}\left(\mathrm{~W}^{\mathrm{c}}\right)\right)^{\mathrm{c}}=\mathrm{f}^{-1}(\mathrm{~W})$. Karena $\mathrm{f}^{-1}\left(\mathrm{~W}^{\mathrm{c}}\right)$ terbuka didalam $X$ maka $\mathrm{f}^{-1}(\mathrm{~W})$ tertutup didalam $\mathrm{X}$.

$(\Leftarrow)$ Diketahui $\mathrm{f}^{-1}(\mathrm{~W})$ tertutup didalam $\mathrm{X}$ untuk setiap himpunan tertutup W didalam $\mathrm{Y}$, akan diperlihatkan $\mathrm{f}$ kontinu pada $\mathrm{X}$.

Diambil sebarang himpunan tertutup $\mathrm{W}$ didalam $\mathrm{Y}$, maka $\mathrm{W}^{\mathrm{c}}$ terbuka didalam $\mathrm{Y}$ dan berdasarkan yang diketahui, $\mathrm{f}^{-1}(\mathrm{~W})$ tertutup didalam $\mathrm{X}$ atau $\mathrm{f}^{-1}\left(\mathrm{~W}^{\mathrm{c}}\right)$ terbuka didalam $\mathrm{X}$, sebab $\left(\mathrm{f}^{-1}(\mathrm{~W})\right)^{\mathrm{c}}=\mathrm{f}^{-1}\left(\mathrm{~W}^{\mathrm{c}}\right)$. Jadi $\mathrm{f}^{-1}\left(\mathrm{~W}^{\mathrm{c}}\right)$ terbuka didalam $\mathrm{X}$, untuk setiap himpunan terbuka $\mathrm{W}^{\mathrm{c}}$ didalam $\mathrm{Y}$ dan berdasarkan teorema 9, maka f kontinu.

\section{Teorema 11}

Misalkan X ruang metrik.

Jika $\mathrm{f}$ dan $\mathrm{g}$ kontinu pada $\mathrm{X}$, maka $\mathrm{f}+\mathrm{g}$, fg dan $\mathrm{f} / \mathrm{g}$ kontinu pada $\mathrm{X}$, asalkan $\mathrm{g}(\mathrm{x}) \neq 0$ untuk setiap $\mathrm{x} \in \mathrm{X}$.

Bukti : 


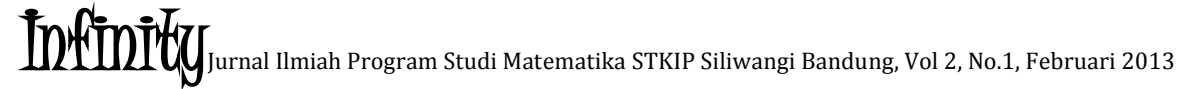

Akan diperlihatkan salah satu saja yaitu $\mathrm{f}+\mathrm{g}$ kontinu dan yang lainnya sejalan. Misalkan $f$ dan $g$ kontinu di $p \in X$, maka berdasarkan teorema $7, \lim _{x \rightarrow p} f(x)=f(p)$ dan $\lim _{x \rightarrow p} g(x)=g(p)$. Jadi $\lim _{x \rightarrow p}(f+g)(x)=\lim _{x \rightarrow p}(f(x)+g(x))$

$$
\begin{aligned}
& =\lim _{x \rightarrow p} f(x)+\lim _{x \rightarrow p} g(x) \\
& =f(p)+g(p)=(f+g)(p) .
\end{aligned}
$$

Jadi $\lim _{x \rightarrow p}(f+g)(x)=(f+g)(x)$ yang berarti $f+g$ kontinu di $p$.

\section{Kekompakan Suatu Fungsi}

Setelah kita membicarakan limit dan kekontinuan fungsi di ruang metrik, selanjutnya kita lihat bagaimana hubungan antara fungsi kontinu dan fungsi kompak.

\section{Definisi 12 (Fungsi Terbatas)}

Misalkan $\mathrm{X}$ ruang metrik, $\mathrm{E} \subset \mathrm{X}$ dan $\mathrm{f}: \mathrm{E} \rightarrow \mathrm{R}$.

f disebut fungsi terbatas jika ada $M>0$ sehingga $|f(x)| \leq M$, untuk setiap $\mathrm{x} \in \mathrm{E}$.

\section{Teorema 13}

Misalkan $\mathrm{X}$ dan $\mathrm{Y}$ ruang metrik, $\mathrm{f}: \mathrm{X} \rightarrow \mathrm{Y}$ dan $\mathrm{X}$ kompak.

Jika f kontinu pada $\mathrm{X}$, maka $\mathrm{f}(\mathrm{X})$ kompak.

\section{Bukti :}

Diambil sebarang selimut terbuka $\left\{\mathrm{V}_{\alpha}\right\}$ untuk $\mathrm{f}(\mathrm{X})$. Berarti $\mathrm{V}_{\alpha}$ merupakan himpunan terbuka didalam $\mathrm{Y}$ untuk setiap $\alpha$. Karena $\mathrm{f}$ kontinu pada $\mathrm{X}$, maka berdasarkan teorema $9, \mathrm{f}^{-1}\left(\mathrm{~V}_{\alpha}\right)$ terbuka didalam $\mathrm{X}$. Akibatnya $\left\{\mathrm{f}^{-1}\left(\mathrm{~V}_{\alpha}\right)\right\}$ merupakan selimut terbuka untuk $X$ dan karena $X$ kompak, maka ada $\alpha_{1}, \alpha_{2}, \ldots, \alpha_{n}$ sehingga

$$
\mathrm{X} \subset \bigcup_{\mathrm{i}=1}^{\mathrm{n}} \mathrm{f}^{-1}\left(\mathrm{~V}_{\alpha \mathrm{i}}\right) \text { atau } \mathrm{f}(\mathrm{X}) \subset \mathrm{f}\left(\bigcup_{\mathrm{i}=1}^{\mathrm{n}} \mathrm{f}^{-1}\left(\mathrm{~V}_{\alpha \mathrm{i}}\right)\right)=\bigcup_{\mathrm{i}=1}^{\mathrm{n}} \mathrm{f}\left(\mathrm{f}^{-1}\left(\mathrm{~V}_{\alpha \mathrm{i}}\right)\right) \subset \bigcup_{\mathrm{i}=1}^{\mathrm{n}} \mathrm{V}_{\alpha \mathrm{i}} \text {. }
$$

Jadi untuk setiap selimut terbuka $\left\{\mathrm{V}_{\alpha}\right\}$ ada $\alpha_{1}, \alpha_{2}, \ldots, \alpha_{\mathrm{n}}$ sehingga $\mathrm{f}(\mathrm{X}) \subset \bigcup_{\mathrm{i}=1}^{\mathrm{n}} \mathrm{V}_{\alpha \mathrm{i}}$ yang berarti $\mathrm{f}(\mathrm{X})$ kompak. 


\section{Teorema 14}

Misalkan $\mathrm{X}$ dan $\mathrm{Y}$ ruang metrik, $\mathrm{f}: \mathrm{X} \rightarrow \mathrm{Y}, \mathrm{X}$ kompak dan $\mathrm{f}$ fungsi satu-satu dan kontinu. Jika $\mathrm{f}^{-1}$ fungsi invers dari $\mathrm{Y}$ ke $\mathrm{X}$ yang didefinisikan $\mathrm{f}^{-1}(\mathrm{f}(\mathrm{x}))=\mathrm{x}$, untuk setiap $\mathrm{x} \in \mathrm{X}$, maka $\mathrm{f}^{-1}$ kontinu.

\section{Bukti :}

Diketahui f kontinu pada $\mathrm{X}$, maka $\mathrm{f}^{-1}(\mathrm{~V})$ terbuka didalam $\mathrm{X}$, untuk setiap himpunan terbuka $\mathrm{V}$ didalam $\mathrm{Y}$. Akan diperlihatkan $\mathrm{f}^{-1}$ kontinu pada $\mathrm{Y}$, artinya cukup diperlihatkan $\mathrm{f}(\mathrm{V})$ terbuka didalam $\mathrm{Y}$, untuk setiap himpunan terbuka V didalam $\mathrm{X}$.

Diambil sebarang himpunan terbuka $\mathrm{V}$ didalam $\mathrm{X}$, maka $\mathrm{V}^{\mathrm{c}}$ tertutup didalam $\mathrm{X}$. Karena $\mathrm{X}$ kompak, maka $\mathrm{V}^{\mathrm{c}}$ kompak dan karena $\mathrm{f}$ kontinu, maka berdasarkan teorema 13, $\mathrm{f}\left(\mathrm{V}^{\mathrm{c}}\right)$ kompak. Berdasarkan teorema Heine Borel maka $\mathrm{f}\left(\mathrm{V}^{\mathrm{c}}\right)$ tertutup. Selanjutnya berdasarkan yang diketahui f fungsi satu-satu, maka $f\left(V^{c}\right)=(f(V))^{c}$ atau $\left(f\left(V^{c}\right)\right)^{c}=f(V)$. Padahal $f\left(V^{c}\right)$ tertutup di dalam $Y$, maka $f(V)$ terbuka didalam $Y$. Jadi untuk sebarang himpunan terbuka $V$ didalam $X$ diperoleh $f(V)$ terbuka didalam Y. Berdasarkan teorema 9, maka $^{-1}$ kontinu pada $\mathrm{Y}$.

\section{Kontinu Seragam}

Pada bagian terakhir, kita lihat bagaimana hubungan antara fungsi kompak dengan fungsi kontinu seragam.

\section{Definisi 15}

Misalkan X dan Y ruang metrik, $\mathrm{f}: \mathrm{X} \rightarrow \mathrm{Y}$.

f disebut kontinu seragam pada $X$ jika untuk setiap $\varepsilon>0$ ada $\delta>0$ sehingga untuk setiap $\mathrm{p}, \mathrm{q} \in \mathrm{X}$ dengan $\mathrm{d}_{\mathrm{X}}(\mathrm{p}, \mathrm{q})<\delta$ berlaku $\mathrm{d}_{\mathrm{Y}}(\mathrm{f}(\mathrm{p}), \mathrm{f}(\mathrm{q}))<\varepsilon$.

Selanjutnya, setiap fungsi yang kontinu seragam adalah kontinu.

\section{Teorema 16}

Misalkan $\mathrm{X}$ dan $\mathrm{Y}$ ruang metrik, $\mathrm{X}$ kompak dan $\mathrm{f}: \mathrm{X} \rightarrow \mathrm{Y}$.

Jika f kontinu pada $\mathrm{X}$, maka $\mathrm{f}$ kontinu seragam pada $\mathrm{X}$.

\section{Bukti :}

Diberikan $\varepsilon>0$ sebarang.

Karena $\mathrm{f}$ kontinu pada $\mathrm{X}$, maka $\mathrm{f}$ kontinu disetiap titik $\mathrm{p} \in \mathrm{X}$, artinya ada $\delta_{\mathrm{p}}>0$ sehingga untuk setiap $\mathrm{q} \in \mathrm{X}$ dengan $\mathrm{d}_{\mathrm{X}}(\mathrm{p}, \mathrm{q})<\delta_{\mathrm{p}}$ berlaku $\mathrm{d}_{\mathrm{Y}}(\mathrm{f}(\mathrm{p}), \mathrm{f}(\mathrm{q}))<\varepsilon / 2$. 
Misalkan $J(p)=\left\{q \in X: d_{X}(p, q)<\frac{1}{2} \delta_{p}\right\}$, maka $\{J(p): p \in X\}$ merupakan selimut terbuka untuk $X$ dan karena $X$ kompak, maka ada $p_{1}, p_{2}, \ldots, p_{n}$ sehingga $\mathrm{X} \subset \bigcup_{\mathrm{i}=1}^{\mathrm{n}} \mathrm{J}\left(\mathrm{p}_{\mathrm{i}}\right)$. Diambil $\delta=\frac{1}{2} \min \left\{\delta_{\mathrm{p} 1}, \delta_{\mathrm{p} 1}, \ldots \delta_{\mathrm{pn}}\right\}$, maka jelas $\delta>0$.

Selanjutnya diambil $\mathrm{p}, \mathrm{q} \in \mathrm{X}$ dengan $\mathrm{d}_{\mathrm{X}}(\mathrm{p}, \mathrm{q})<\delta$, maka $\mathrm{p} \in \mathrm{J}\left(\mathrm{p}_{\mathrm{m}}\right)$ untuk suatu $\mathrm{m}$, $1 \leq \mathrm{m} \leq \mathrm{n}$ dan diperoleh $\mathrm{d}_{\mathrm{X}}\left(\mathrm{p}, \mathrm{p}_{\mathrm{m}}\right)<\frac{1}{2} \delta_{\mathrm{pm}}<\delta_{\mathrm{pm}}$. Akibatnya $\mathrm{d}_{\mathrm{Y}}\left(\mathrm{f}(\mathrm{p}), \mathrm{f}\left(\mathrm{p}_{\mathrm{m}}\right)<\varepsilon / 2\right.$. Lebih lanjut $\mathrm{d}_{\mathrm{x}}\left(\mathrm{p}_{\mathrm{m}}, \mathrm{q}\right) \leq \mathrm{d}_{\mathrm{X}}\left(\mathrm{p}_{\mathrm{m}}, \mathrm{p}\right)+\mathrm{d}_{\mathrm{x}}(\mathrm{p}, \mathrm{q})<\frac{1}{2} \delta_{\mathrm{pm}}+\delta<\frac{1}{2} \delta_{\mathrm{pm}}+\frac{1}{2} \delta_{\mathrm{pm}}=\delta_{\mathrm{pm}}$. Akibatnya $\mathrm{d}_{\mathrm{Y}}\left(\mathrm{f}\left(\mathrm{p}_{\mathrm{m}}\right), \mathrm{f}(\mathrm{q})<\varepsilon / 2\right.$.

Jadi untuk setiap $\varepsilon>0$, ada $\delta>0$ sehingga untuk setiap $\mathrm{p}, \mathrm{q} \in \mathrm{X}$ dengan $\mathrm{d}_{\mathrm{X}}(\mathrm{p}, \mathrm{q})<\delta$ berlaku $d_{\mathrm{Y}}(\mathrm{f}(\mathrm{p}), \mathrm{f}(\mathrm{q})) \leq \mathrm{d}_{\mathrm{Y}}\left(\mathrm{f}(\mathrm{p}), \mathrm{f}\left(\mathrm{p}_{\mathrm{m}}\right)\right)+\mathrm{d}_{\mathrm{Y}}\left(\mathrm{f}\left(\mathrm{p}_{\mathrm{m}}\right), \mathrm{f}(\mathrm{q})\right)<\varepsilon / 2+\varepsilon / 2=\varepsilon$. Hal ini berarti $\mathrm{f}$ kontinu seragam pada $\mathrm{X}$.

\section{Kesimpulan}

1. Pengertian limit fungsi dan kekontinuan fungsi pada ruang metrik sama dengan pengertian limit fungsi dan kekontinuan fungsi pada $\mathrm{R}$ di kalkulus, hanya bedanya kalau di kalkulus yang dimaksud metrik/jarak adalah nilai mutlak, sedangkan di sini adalah jarak yang umum yang memenuhi definisi metrik.

2. Fungsi $\mathrm{f}: \mathrm{X} \rightarrow \mathrm{Y}$ kontinu pada $\mathrm{X}$ jika dan hanya jika $\mathrm{f}^{-1}(\mathrm{~V})$ terbuka didalam $\mathrm{X}$, untuk setiap himpunan terbuka $\mathrm{V}$ didalam $\mathrm{Y}$.

3. Fungsi $f$ kontinu pada $X$ jika dan hanya jika $f^{-1}(W)$ tertutup didalam $X$, untuk setiap himpunan tertutup $\mathrm{W}$ didalam $\mathrm{Y}$.

4. Jika fungsi $f$ kontinu pada ruang metrik $X$ yang kompak maka $f(X)$ kompak.

5. Jika fungsi $f$ kontinu pada ruang metrik $X$ yang kompak maka $f$ kontinu seragam pada X.

\section{DAFTAR PUSTAKA}

Apostol, T.M. (1974). Mathematical Analysis (Second Edition). Addison-Wesley Publishing Company, Inc. Philippines.

Munkres, J.R. (1975). Topologi (A First Course). Prentice-Hall, Inc, Englewood Cliffs, New Jersey. USA.

Rudin, Walter (1976). Principles of Mathematical Analysis (Third Edition). McGraw-Hill. Singapore. 